\title{
Prenatal SSRI Use and Offspring With Autism Spectrum Disorder or Developmental Delay
}

AUTHORS: Rebecca A. Harrington, PhD, MPH, ${ }^{a}$ Li-Ching Lee, PhD, ScM, ${ }^{a}$ Rosa M. Crum, MD, MHS, ${ }^{b}$ Andrew W. Zimmerman, MD, ${ }^{c}$ and Irva Hertz-Picciotto, PhD, MPH

aDepartment of Epidemiology, Johns Hopkins Bloomberg School of Public Health, and ${ }^{b}$ Departments of Epidemiology, Psychiatry, and Mental Health, Johns Hopkins Medical Institutions,

Baltimore, Maryland; ' ${ }^{D}$ Department of Pediatrics, University of Massachusetts Medical School, Worcester, Massachusetts; and dDepartment of Public Health Sciences and the MIND Institute, University of California, Davis, Davis, California

\section{KEY WORDS}

selective serotonin reuptake inhibitors, autism, developmental delay, pregnancy, epidemiology

\section{ABBREVIATIONS}

ADI-R-Autism Diagnostic Interview-Revised

ADOS—Autism Diagnostic Observation Schedule

ASD—autism spectrum disorder

CHARGE-Childhood Autism Risks from Genetics and the

Environment

Cl-confidence interval

$\mathrm{DD}$-developmental delay

$\mathrm{OR}$ - Odds ratio

SCQ-Social Communication Questionnaire

SSRI—selective serotonin reuptake inhibitor

TD—typical development

Dr Harrington conceptualized and designed the selective serotonin reuptake inhibitor (SSRI) study, acquired data, carried out the initial analyses, interpreted data, and drafted and revised the initial manuscript; Dr Lee conceptualized and designed the SSRI study, interpreted data, and reviewed and revised the manuscript; Drs Crum and Zimmerman interpreted data and reviewed and revised the manuscript; Dr HertzPicciotto conceptualized, designed, and obtained funding for the Childhood Autism Risks from Genetics and the Environment (CHARGE) Study, acquired and interpreted data, and reviewed and revised the manuscript; and all authors approved the final manuscript as submitted.

www.pediatrics.org/cgi/doi/10.1542/peds.2013-3406

doi:10.1542/peds.2013-3406

Accepted for publication Feb 14, 2014

Address correspondence to Rebecca A. Harrington, PhD, MPH, Johns Hopkins Bloomberg School of Public Health, 615 North Wolfe St, Suite E6037, Baltimore, MD 21205. E-mail: rharring@jhsph.edu

PEDIATRICS (ISSN Numbers: Print, 0031-4005; Online, 1098-4275). Copyright (c) 2014 by the American Academy of Pediatrics

(Continued on last page)
WHAT'S KNOWN ON THIS SUBJECT: Serotonin is critical in early brain development, creating concerns regarding prenatal exposure to factors influencing serotonin levels, like selective serotonin reuptake inhibitors (SSRIS). Prenatal SSRI use was recently associated with autism; however, its association with other developmental delays is unclear.

WHAT THIS STUDY ADDS: This population-based case-control study in young children provides evidence that prenatal SSRI use may be a risk factor for autism and other developmental delays. However, underlying depression and its genetic underpinnings may be a confounder.

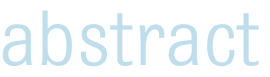

OBJECTIVE: To examine associations between prenatal use of selective serotonin reuptake inhibitors (SSRIs) and the odds of autism spectrum disorders (ASDs) and other developmental delays (DDs).

METHODS: A total of 966 mother-child pairs were evaluated (492 ASD, 154 DD, 320 typical development [TD]) from the Childhood Autism Risks from Genetics and the Environment (CHARGE) Study, a populationbased case-control study. Standardized measures confirmed developmental status. Interviews with biological mothers ascertained prenatal SSRI use, maternal mental health history, and sociodemographic information.

RESULTS: Overall, prevalence of prenatal SSRI exposure was lowest in TD children (3.4\%) but did not differ significantly from ASD (5.9\%) or DD (5.2\%) children. Among boys, prenatal SSRI exposure was nearly 3 times as likely in children with ASD relative to TD (adjusted odds ratio [OR]: 2.91; 95\% confidence interval [CI]: 1.07-7.93); the strongest association occurred with first-trimester exposure (OR: 3.22; $95 \% \mathrm{Cl}$ : 1.17-8.84). Exposure was also elevated among boys with DD (OR: 3.39; $95 \% \mathrm{Cl}: 0.98-11.75)$ and was strongest in the third trimester (OR: 4.98; 95\% Cl: 1.20-20.62). Findings were similar among mothers with an anxiety or mood disorder history.

CONCLUSIONS: In boys, prenatal exposure to SSRIs may increase susceptibility to ASD or DD. Findings from published studies on SSRIs and ASD continues to be inconsistent. Potential recall bias and residual confounding by indication are concerns. Larger samples are needed to replicate DD results. Because maternal depression itself carries risks for the fetus, the benefits of prenatal SSRI use should be carefully weighed against potential harms. Pediatrics 2014;133:e1241-e1248 
Autism spectrum disorders (ASDs) are estimated to affect 1 in 88 children, ${ }^{1}$ and the prevalence continues to increase. Hyperserotonemia is found in approximately one-third of children with autism. ${ }^{2}$ Altered serotonin levels during early development are speculated to lead to abnormal brain circuitry and autism symptoms. ${ }^{3}$ Selective serotonin reuptake inhibitors (SSRIs), which increase extracellular serotonin, are the most common antidepressants prescribed during pregnancy: $\sim 4 \%$ of pregnant women use an SSRI, and use has increased over time. ${ }^{4}$ Given the central role of serotonin in brain development through maternal-fetal and placental interactions, 5,6 potential long-term developmental effects from prenatal SSRI exposure should be assessed.

Animal models of autism using serotonin agonists and medications that increase serotonin ${ }^{3,7}$ support the notion that during early gestation, high circulating serotonin reaches the fetal brain, causing loss of serotonin nerve terminals. ${ }^{3}$ Conversely, SSRIs and similaracting medications could be protective by re-regulating maternal serotonin levels. In mice, maternal fluoxetine in early development reversed the effects of prenatal maternal stress on depressive-like behaviors and hippocampal neurogenesis in adolescent offspring. ${ }^{8}$

In humans, SSRIs cross the placenta, ${ }^{9}$ reduce serotonin reuptake in the placenta ${ }^{10}$ and fetus, ${ }^{11}$ and may reduce uterine blood flow, leading to fetal hypoxemia. ${ }^{12}$ Fetal hypoxia can adversely affect birth and developmental outcomes; an association with ASD is plausible. $^{13}$ Recently, 2 large casecontrol studies using medical records/ registries reported elevated risk of ASD with prenatal SSRI exposure, ${ }^{14,15}$ whereas a registry-based cohort study reported no association. ${ }^{16}$ Building on previous literature, we used a population-based study in which diagnoses of ASD and other developmental delays (DDs) were confirmed by standardized instruments to investigate prenatal SSRI use.

\section{METHODS}

Participants were families enrolled in the Childhood Autism Risks from Genetics and the Environment (CHARGE) Study. ${ }^{17}$ This population-based casecontrol investigation recruited children with ASD, with DDs other than ASD, and from the general population. CHARGE eligibility criteria included being 2 to 5 years old, born in California, having a parent who speaks English or Spanish, and living with at least 1 biological parent and in the study catchment area of specified California Regional Centers, which coordinate services for persons with developmental disabilities.

Children with ASD and DD were identified from those qualifying for services through California's Department of Developmental Services, provider referrals, public outreach, and selfreferrals. Population controls were identified by using state birth files and were frequency matched to autism cases by age, gender (targeted 4:1 male:female ratio), and regional center. CHARGE was approved by institutional review boards at the University of California, Davis; the University of California, Los Angeles; and the State of California. Written informed consent was obtained. Clinical assessments and standardized interviews were conducted in English or Spanish by trained staff with reliability on the respective instruments. This analysis includes CHARGE enrollees between April 2003 and August 2010.

To confirm ASD diagnoses, children were assessed with the Autism Diagnostic Interview-Revised (ADI-R) ${ }^{18}$ and the Autism Diagnostic Observation Schedule (ADOS). ${ }^{19}$ A final autism di- agnosis required meeting criteria on the communication, social interaction, and repetitive behavior domains of the ADI-R and scoring at or above the social plus communication cutoff for autism on the ADOS. ASD was defined by using the criteria of Risi et al. ${ }^{20}$ Children with DD diagnosed by regional centers and general population controls were screened for autism by using the Social Communication Questionnaire (SCQ) ${ }^{21}$ The ADI-R and ADOS were administered for children who screened positive (SCQ $\geq 15$ ), and if they met criteria, were reclassified as ASD. The Vineland Adaptive Behavior Scales ${ }^{22}$ and Mullen Scales of Early Learning23 were used to define DD (composite score $\leq 70$ on either scale). The typical development (TD) group comprised children recruited from the general population scoring $>69$ on the Mullen Scales of Early Learning and Vineland Adaptive Behavior Scales and $<15$ on the SCQ. Siblings of probands were excluded, regardless of case status. The final sample included 966 mother-child pairs: 492 ASD, 154 DD, and 320 TD.

A standardized telephone interview was conducted with the child's biological mother to collect exposures for an index period covering 3 months before conception to the end of pregnancy or through breastfeeding. Mothers who reported having a medical condition or taking medication, including SSRIs, during the index period were asked to specify timing in monthly intervals. Information was also collected on the following: maternal age, education, and birthplace; maternal use during pregnancy of nicotine, alcohol, illicit substances, non-SSRI medications (eg, tricyclic antidepressants), and supplements that affect serotonin (eg, St John's wort); maternal obesity, diabetes, or hypertension; mother's anxiety/mood disorder history; and child's year of birth and race. Prenatal medical records were used, when 
available, to supplement self-reported mental health history and for crosssource comparison of self-reported SSRI use. Preterm birth and low birth weight were analyzed but not included in multivariate models because they were potentially affected by SSRIs, the adjustment for which could introduce bias. $^{24}$

Adjusted odds ratios (ORs) and 95\% confidence intervals (Cls) were estimated by using logistic regression. Inverse probability weights were used in all models to address differential participation and to obtain results generalizable to the study's base population. Two models were constructed with SSRI use in pregnancy as the exposure: ASD versus TD and DD versus TD. We fit separate models for SSRI use during each trimester relative to no use during pregnancy. To address confounding, covariates were included in the models if associated with both SSRI exposure and diagnostic group (OR $\geq 2.0$ or $P<.20$ ) and not an intermediate between exposure and outcome. Final models included child's birth year (1998-2003, 2004-2007), regional center, and mother's birthplace (United States, Mexico, or elsewhere). We also stratified by child gender to examine effect modification. To separate effects of maternal mental health status from those of SSRI use, we then restricted analyses to mothers who experienced an anxiety or mood disorder at any time before their child's birth.

Prenatal medical record information was compared with self-reported SSRI use during pregnancy to assess reporting bias. Intermethod reliability was calculated (percentage of agreement and $\kappa$ ). Outside limits of agreement were generated by recoding unavailable medical records to either concordant or discordant with selfreport. Neither self-report nor medical records represent a gold standard.
Self-report depends on recall, whereas prenatal medical records may underor overreport: prenatal records may not capture SSRI prescriptions from other providers, and not all prescriptions are filled and taken. These statistics therefore measure reliability, not validity. Analyses were conducted by using Stata/SE 11.2 (StataCorp LP, College Station, TX).

\section{RESULTS}

Table 1 presents study population characteristics. The mean age of enrolled children was 3.7 (SD: 0.81) years. TD children were slightly younger $(3.6$ years) than children with ASD (3.8 years; $P<.001$ ) or DD (3.8 years; $P<$ .001). ASD and TD children had similar birth weight and preterm delivery and maternal socioeconomic characteristics. DD children were more likely to be female (because they were not matched), Hispanic, to have low birth weight, to have mothers born in Mexico, to have a lower education, and use public health insurance. Two-thirds of the sample was born between 1998 and 2003, before the Food and Drug Administration began changes to SSRI labeling, calling into question the safety of prenatal SSRI use. A greater proportion of ASD children were born before 2004 compared with DD ( $P=$ .001) and TD children $(P<.001)$. No mothers born outside the United States reported SSRI use during pregnancy.

Forty-eight women (5\%) reported prenatal SSRI use. Of these, $44 \%$ took fluoxetine, $21 \%$ sertraline, $19 \%$ paroxetine, $8 \%$ citalopram, and $8 \%$ escitalopram. Among SSRI users, 5 also reported using a non-SSRI antidepressant, 2 an anxiolytic, and 1 an antipsychotic. In unadjusted analyses (Table 2), prenatal SSRI use was lowest among TD mothers, but not significantly so in boys and girls combined. Among boys, prenatal SSRI use was higher in ASD compared with TD mothers $(P=.04)$, and use during the third trimester was higher among both ASD and DD compared with TD mothers ( $P=.03$ for both). For girls, small cell sizes precluded analysis.

Among mothers whose mental health history was available (440 ASD, 140 DD, 300 TD), more than one-quarter whose child had ASD (28\%) or DD (29\%) had a lifetime history of anxiety or mood disorder before the child's birth, as compared with $22 \%$ in the TD group $(P=.19)$ (data not shown). Of mothers with a history of anxiety or mood disorder, 20\% reported SSRI use during pregnancy. Two with SSRI use during pregnancy had no anxiety or mood disorder history. Within the anxiety/ mood disorder subset, prenatal SSR use did not differ significantly by child's diagnosis, overall or in boys.

In multivariate analyses (Table 3) involving the total sample or the subset of mothers with anxiety/mood disorder history, children exposed to SSRIs during pregnancy were no more likely to have an ASD or DD than were unexposed children. However, among boys, prenatal exposure to SSRIs nearly tripled the association with ASD (OR: 2.92; 95\% Cl: 1.07-7.93), and more than tripled it for DD (OR: $3.39 ; 95 \% \mathrm{Cl}$ 0.98-11.75). The highest association for ASD was with first-trimester exposure, whereas the association with DD was elevated in the second and third trimesters, although few DD children were exposed. The pattern was similar among boys in the anxiety/mood disorder subset, but with low precision (102 ASD, 29 DD, 50 TD). Adjusting, rather than restricting, for maternal mental health yielded similar findings.

Possible exposure misclassification of first-trimester use was examined by considering women who reported stopping SSRI use the month before conception as exposed during the first trimester. Results were similar, although attenuated. We also examined breastfeeding as a potential 
confounder; however, it was not associated with prenatal SSRI exposure, and therefore was unlikely to confound study results.

0verall percentage agreement between self-reported and prenatal medical record report of SSRI use during pregnancy was $96.7 \%$, with $\kappa=0.66$. These statistics were highest for the DD group (98.2\% agreement, $\kappa=0.85$ ) compared with ASD and TD groups (95.8\% and $97.4 \%$ agreement, respectively; both $\kappa=0.61$ ) (Table 4). A standard interpretation of $\kappa$ is that 0.61 to 0.80 reflects substantial agreement, whereas 0.81 to 1.00 is almost perfect. ${ }^{25}$

\section{DISCUSSION}

In this population-based case-control study, mothers' reported use of SSRIs during pregnancy was associated with increased risk of ASD and DD in boys. Too few girls were exposed for analysis. Results were robust to sensitivity analyses and adjustment for confounders. Trimester-specific analyses in boys showed the greatest association with ASD during first-trimester SSRI exposure. Among the subset whose mothers previously had an anxiety or mood disorder, the association was also elevated among boys, although not significantly. Thirdtrimester exposure conveyed a 5 -fold higher association with DD. The small numbers of exposed DD cases and exposed TD controls resulted in low precision.

Although we had few girls, the substantially stronger effect in boys alone compared with the combined analysis suggests possible effect modification by gender. Serotonergic function is influenced by sex hormones, ${ }^{26,27}$ and serotonin system biological variations by gender have been reported. ${ }^{28}$ Mice pups prenatally exposed to agents that can alter serotonergic function, including an SSRI, ${ }^{29,30}$ showed perma- nent sex-specific changes in behavior. Moreover, in children exposed prenatally to antidepressants, delays in motor milestones were greater in boys. ${ }^{31}$

An obstacle to drawing inferences about prenatal SSRI exposure is the difficulty in isolating SSRIs' effects from those of their indications for use. This problem is underscored by aggregation of psychiatric conditions in relatives of children with autism. ${ }^{32} \mathrm{Re}$ liance on medical records to assess mental health history ${ }^{14,15}$ is problematic because mothers who do not seek services are missed, and mental health conditions may be underreported in medical records. ${ }^{33}$ However, underreporting of depression may also occur with maternal recall. ${ }^{34}$ Studies adjusting for maternal mental health ascertained from either source may therefore overestimate psychotropic medication effects.

Studies of prenatal SSRI exposure with infant and child development generally indicate no adverse effects, ${ }^{35}$ except for a few reports of gross motor development deficits. 31,36,37 Limitations in this literature include small samples and insufficient control for maternal mental health. Furthermore, whether observed differences predict persistent impairment is unknown. Recent ASD studies ${ }^{14-16}$ using electronic medical records and registries included large samples of pregnant women with prospectively collected medication use. However, ASD diagnoses were not validated. Furthermore, studies by Croen et al ${ }^{14}$ using Kaiser patients and by Hviid et al ${ }^{16}$ using Danish registries were unable to confirm whether dispensed SSRIs were actually taken by

TABLE 1 Demographic and Perinatal Characteristics of Children With ASD, DD, and TD

\begin{tabular}{|c|c|c|c|c|}
\hline Characteristic & $\operatorname{ASD}(n=492)$ & $\mathrm{DD}(n=154)$ & $\mathrm{TD}(n=320)$ & $P$ \\
\hline \multicolumn{5}{|l|}{ Children } \\
\hline Age & & & & $.002^{\star \S}$ \\
\hline 2 years & $104(21.1)$ & $25(16.2)$ & $98(30.6)$ & \\
\hline 3 years & $178(36.2)$ & $61(39.6)$ & $114(35.6)$ & \\
\hline $4-5$ years & $210(42.7)$ & $68(44.2)$ & $108(33.8)$ & \\
\hline Male gender & $421(82.5)$ & $101(65.6)$ & $264(85.6)$ & $<.001^{\dagger \S}$ \\
\hline Race/ethnicity & & & & .20 \\
\hline Non-Hispanic white & $247(50.2)$ & $64(41.6)$ & $165(51.6)$ & \\
\hline Non-Hispanic black, Asian, or multiracial & $90(18.3)$ & $28(18.2)$ & $60(18.8)$ & \\
\hline Hispanic & $155(31.5)$ & $62(40.3)$ & $95(29.7)$ & \\
\hline Year of birth & & & & $<.001^{\star \dagger}$ \\
\hline 1998-2003 & $352(71.5)$ & $84(54.6)$ & $193(60.3)$ & \\
\hline 2004-2007 & $140(28.5)$ & $70(45.5)$ & $127(39.7)$ & \\
\hline Low birth weight (<2500 g) & $36(7.3)$ & $21(13.6)$ & $20(6.3)$ & $.03^{\dagger \S}$ \\
\hline Preterm birth ( $<37$ weeks) & $51(10.4)$ & $20(13.0)$ & $33(10.3)$ & .64 \\
\hline \multicolumn{5}{|l|}{ Mothers } \\
\hline Age & & & & .13 \\
\hline$<25$ years & $70(14.2)$ & $33(21.4)$ & $56(17.5)$ & \\
\hline $25-34$ years & $287(58.3)$ & $79(51.3)$ & $191(59.7)$ & \\
\hline$\geq 35$ years & $135(27.4)$ & $42(27.3)$ & $73(22.8)$ & \\
\hline Education & & & & $<.001^{\star \dagger \S}$ \\
\hline$\leq$ High school & 68 (13.8) & $43(27.9)$ & $51(15.9)$ & \\
\hline Some college & $200(40.7)$ & $65(42.2)$ & $101(31.6)$ & \\
\hline$\geq$ College degree & $224(45.5)$ & $46(29.9)$ & $168(52.5)$ & \\
\hline Birthplace & & & & $.001^{\dagger \S}$ \\
\hline United States & $370(75.2)$ & $118(76.6)$ & $258(80.6)$ & \\
\hline Mexico & $41(8.3)$ & $25(16.2)$ & $22(6.9)$ & \\
\hline Other & $81(16.5)$ & $11(7.1)$ & $40(12.5)$ & \\
\hline Private insurance & $400(81.3)$ & $108(70.1)$ & $276(86.5)$ & $<.001^{\star \dagger \S}$ \\
\hline
\end{tabular}


mothers, whereas the Rai et al ${ }^{15}$ study from Stockholm, Sweden, only included self-reported use at the first antenatal visit (median of 10 weeks' gestation). The Kaiser and Swedish studies reported modestly increased ASD risk among children prenatally exposed to SSRIs, independent of maternal mental health, whereas no significant differences were observed in the Danish cohort. Similar to our results, the Kaiser study found the highest risk with first-trimester use. The Swedish study could not assess timing of use. Neither assessed differences in SSRI effects by child gender.

Similar to the Danish cohort results, we found no association when boys and girls were combined. SSRI use by CHARGE study participants during pregnancy only revealed significant associations with ASD among boys. Hviid et al ${ }^{16}$ reported no data on gender, nor gender-stratified analyses. The authors also did not assess effect modification by indication; their larger number of exposed cases might have permitted an informative analysis among mothers with depression and provided evidence on the replicability large Danish cohort, the prevalence of prenatal SSRI use was much lower of results from Sweden. ${ }^{15}$ Despite the

than in our study, as was the prevalence in Sweden, raising questions about generalizability and whether prenatal SSRI users in Scandinavia are a more select group than in the United States.

Strengths of our study include its relatively large sample of cases, population-based controls, and a uniform protocol to confirm ASD and DD diagnoses by trained clinicians using validated standardized instruments. Different from the 3 previous reports, ours is the first to examine a DD group. We adjusted for selection bias and systematically assessed and controlled for confounders, including maternal mental health. The collection of exposure data via maternal recall has both strengths and weaknesses. A strength is that one can capture whether SSRIs were actually taken by the mother, rather than simply dispensed. A limitation is potential recall bias. Some evidence supports accurate recall for psychotropic medication use during pregnancy in women with a history of mental health problems. ${ }^{34}$ One study found that, compared with antenatal medical records, maternal recall 4 to 9 years after childbirth was very good for the majority of outcomes examined and generally did not vary by maternal

TABLE 2 Unadjusted Prevalence of SSRI Use During Pregnancy

\begin{tabular}{|c|c|c|c|c|c|c|}
\hline \multirow[t]{2}{*}{ SSRI Use } & \multicolumn{3}{|c|}{ Boys and Girls } & \multicolumn{3}{|c|}{ Boys 0nly } \\
\hline & ASD & DD & TD & ASD & DD & TD \\
\hline \multicolumn{7}{|l|}{ Total sample } \\
\hline$n$ & 492 & 154 & 320 & 421 & 101 & 264 \\
\hline Pregnancy, $n$ (\%) & $29(5.9)$ & $8(5.2)$ & $11(3.4)$ & $25(5.9)^{*}$ & $6(5.9)$ & $7(2.7)^{*}$ \\
\hline Trimester 1 & $21(4.3)$ & $4(2.6)$ & $10(3.1)$ & $18(4.3)$ & $4(4.0)$ & $6(2.8)$ \\
\hline Trimester 2 & $16(3.3)$ & $7(4.6)$ & $7(2.2)$ & $14(3.3)$ & $5(5.0)$ & $4(1.5)$ \\
\hline Trimester $3^{\mathrm{a}}$ & $20(4.1)$ & $8(5.3)$ & $7(2.2)$ & $18(4.3)^{\star}$ & $6(6.0)^{\S}$ & $4(1.5)^{\star \S}$ \\
\hline \multicolumn{7}{|c|}{ Anxiety/mood disorder history subset ${ }^{\mathrm{b}}$} \\
\hline$n$ & 122 & 40 & 67 & 102 & 29 & 50 \\
\hline Pregnancy, $n$ (\%) & $28(23)$ & $8(20)$ & $10(15)$ & $24(24)$ & $6(21)$ & $6(12)$ \\
\hline Trimester 1 & $21(17)$ & $4(10)$ & $9(13)$ & $18(18)$ & $4(14)$ & $5(10)$ \\
\hline Trimester 2 & $16(13)$ & $7(18)$ & $7(11)$ & $14(14)$ & $5(17)$ & $4(8)$ \\
\hline Trimester $3^{\mathrm{a}}$ & $19(16)$ & $8(20)$ & $7(11)$ & $17(17)$ & $6(21)$ & $4(8)$ \\
\hline
\end{tabular}

$P$ values were calculated by using likelihood ratio $\chi^{2}$ tests. Significant pairwise group differences $(P \leq .05)$ : *ASD versus TD, ${ }^{\S}$ DD versus TD. ASD versus DD did not differ for any comparison.

a Preterm delivery led to there being no third trimester for 3 participants in the ASD group (all boys), 2 participants in the DD group (1 boy, 1 girl) in the total sample, and for 1 participant in the ASD group (boy) in the subset.

${ }^{\mathrm{b}}$ Due to limited precision in the subgroup, percentages are reported without decimals. characteristics or child behavior problems. ${ }^{38}$ We found cases and controls were similar in the level of discrepancy between self-reported and prenatal medical record SSRI use, suggesting very little recall bias. Moreover, some discrepancies between self-report and medical records in our sample could be due to use of prenatal records for comparison. Had other maternal medical records been available (eg, primary care and mental health), more of the positive self-reports might have been confirmed. However, the impact of additional medical record information on measures of agreement would be unlikely to vary by case status.

Other potential limitations are of note. First, residual confounding by indication for SSRI use remains possible because we were unable to assess the severity of mental health symptoms. Second, lack of data on SSRI dosage precluded dose-response analyses. However, dosage may not correlate well with circulating SSRI levels, given differences in metabolism arising from, for example, metabolic gene polymorphisms. ${ }^{39}$ Furthermore, the usually effective minimum dose of each SSRI produces comparable effects on the degree of serotonin reuptake inhibition, a surrogate for efficacy. ${ }^{40}$ Third, assessment of individual SSRI formulations was unfeasible, because few women used SSRIs during pregnancy. Fourth, the relatively small sample of DD children resulted in imprecise estimates of association, which should be viewed with caution. The interpretation of findings in the DD group also should be tempered because this heterogeneous group included known genetic or chromosomal syndromes. Because of prenatal and neonatal screening tests, some mothers were likely aware of their child's genetic make-up, which might have led some to use SSRIs. Finally, with regard to possible chance associations, in our 
TABLE 3 Estimated Risk for ASD and DD Associated With Maternal Reported SSRI Use During Pregnancy

\begin{tabular}{|c|c|c|c|c|}
\hline & \multicolumn{2}{|c|}{ Boys and Girls } & \multicolumn{2}{|c|}{ Boys Only } \\
\hline & Unadjusted & Adjusted $^{a}$ & Unadjusted & Adjusted $^{\mathrm{a}}$ \\
\hline \multicolumn{5}{|c|}{ Total sample $(N=966)$} \\
\hline \multicolumn{5}{|c|}{ ASD versus TD } \\
\hline Pregnancy & $1.52(0.65-3.53)$ & $1.55(0.59-4.08)$ & $2.68(1.07-6.73)^{*}$ & $2.92(1.07-7.93)^{\star}$ \\
\hline Trimester 1 & $1.32(0.53-3.34)$ & $1.38(0.48-4.01)$ & $2.76(1.04-7.32)^{*}$ & $3.22(1.17-8.84)^{*}$ \\
\hline Trimester 2 & $0.85(0.30-2.42)$ & $0.89(0.24-3.24)$ & $1.73(0.53-5.67)$ & $2.25(0.54-9.42)$ \\
\hline Trimester 3 & $1.15(0.41-3.19)$ & $1.18(0.35-4.02)$ & $2.40(0.75-7.65)$ & $2.91(0.76-11.10)$ \\
\hline \multicolumn{5}{|l|}{ DD versus TD } \\
\hline Pregnancy & $2.03(0.68-6.07)$ & $1.62(0.59-4.42)$ & $4.60(1.37-15.43)^{*}$ & $3.39(0.98-11.75)$ \\
\hline Trimester 1 & $1.21(0.32-4.61)$ & $1.05(0.31-3.54)$ & $3.69(0.94-14.46)$ & $3.26(0.84-12.71)$ \\
\hline Trimester 2 & $2.34(0.67-8.19)$ & $1.96(0.64-6.06)$ & $5.87(1.40-24.67)^{*}$ & $4.41(1.01-19.17)^{\star}$ \\
\hline Trimester 3 & $2.68(0.80-9.00)$ & $2.19(0.74-6.49)$ & $6.82(1.71-27.12)^{\star \star}$ & $4.98(1.20-20.62)^{*}$ \\
\hline \multicolumn{5}{|c|}{$\begin{array}{l}\text { Anxiety/mood disorder } \\
\text { history subset }(N=229)\end{array}$} \\
\hline \multicolumn{5}{|c|}{ ASD versus TD } \\
\hline Pregnancy & $1.51(0.59-3.86)$ & $1.86(0.76-4.58)$ & $2.85(0.99-8.19)$ & $3.17(0.91-11.00)$ \\
\hline Trimester 1 & $1.39(0.50-3.84)$ & $1.70(0.66-4.37)$ & $3.25(1.07-9.85)^{*}$ & $3.52(0.93-13.34)$ \\
\hline Trimester 2 & $0.83(0.28-2.47)$ & $1.12(0.40-3.11)$ & $1.67(0.48-5.79)$ & $1.80(0.39-8.37)$ \\
\hline Trimester 3 & $1.05(0.36-3.09)$ & $1.43(0.52-3.95)$ & $2.17(0.64-7.41)$ & $2.45(0.54-11.22)$ \\
\hline \multicolumn{5}{|l|}{ DD versus TD } \\
\hline Pregnancy & $1.79(0.54-5.93)$ & $1.44(0.44-4.75)$ & 3.86 (0.99-14.99) & $2.30(0.55-9.62)$ \\
\hline Trimester 1 & $1.07(0.26-4.51)$ & $0.99(0.26-3.83)$ & $3.27(0.73-14.76)$ & $2.21(0.52-9.39)$ \\
\hline Trimester 2 & $1.92(0.51-7.25)$ & $1.76(0.47-6.60)$ & $4.28(0.93-19.61)$ & $2.62(0.54-12.80)$ \\
\hline Trimester 3 & $2.17(0.60-7.86)$ & $1.88(0.51-6.88)$ & $4.91(1.13-21.37)^{*}$ & $2.83(0.60-13.43)$ \\
\hline
\end{tabular}

Data are presented as 0 Rs $\left(95 \% \mathrm{Cls}\right.$ ) and were weighted to adjust for selection bias. ${ }^{\star} P \leq .05,{ }^{\star \star} P \leq .01$

a Adjusted for regional center, child's year of birth, and birthplace of mother. primary analyses (multivariate adjusted, full sample) we presented 8 associations in both genders combined and found none to be significant $(<1$ would be expected by chance); we additionally presented 8 associations in boys alone, among which 4 were significant, a pattern that did not suggest a random fluctuation.

\section{CONCLUSIONS}

Our data suggest that prenatal exposure to SSRIs may be associated with an increased risk of ASD, at least in boys, with the greatest risk when exposure is during the first trimester. These findings are biologically plausible given that SSRIs interact with the placenta, ${ }^{10}$ may raise maternal serotonin to abnormal levels, and act directly on the fetus. ${ }^{41}$ Serotonin's integral role in fetal brain development includes modulating early neurodevelopmental processes such as cell division, neurite outgrowth, and neuronal migration, ${ }^{5}$ with the first trimester a period of development especially sensitive to alterations in serotonergic functioning. Recent reviews give a more detailed
TABLE 4 Intermethod Reliability by Diagnostic Group

\begin{tabular}{lccc}
\hline SSRI Exposure During Pregnancy & ASD $(n=492)$ & DD $(n=154)$ & TD $(n=320)$ \\
\hline $\begin{array}{l}\text { Self-report/medical records, } n(\%) \\
\quad\end{array}$ & $13(2.6)$ & $6(3.9)$ & $5(1.6)$ \\
Yes/yes & $10(2.0)$ & $2(1.3)$ & $4(1.3)$ \\
Yes/no & $5(1.0)$ & $0(0.0)$ & $2(0.6)$ \\
No/yes & $328(66.7)$ & $103(66.9)$ & $220(68.8)$ \\
No/no & $130(26.4)$ & $43(27.9)$ & $87(27.2)$ \\
No/not available & $6(1.2)$ & $0(0.0)$ & $2(0.6)$ \\
Yes/not available & $95.8(94.3-97.0)$ & $98.2(96.8-98.7)$ & $97.4(93.7-98.1)$ \\
Percentage of agreement (range) & $0.61(0.45-0.70)$ & $0.85(0.69-0.85)$ & $0.61(0.46-0.69)$ \\
$\kappa$ (range) & & &
\end{tabular}

Percentages may not total 100 due to rounding. discussion relating prenatal SSRI use with ASD and other developmental outcomes. ${ }^{42,43}$

Whether the risk of DDs other than ASD is also elevated in association with prenatal SSRI exposure requires confirmation with a larger sample size; research might usefully address how maternal SSRI use is affected by the knowledge of predisposing genetic conditions. Also needed are studies in more girls, sufficient sample sizes to address possible differential effects from specific SSRIs, and attention to mechanisms by which SSRls might influence ASD and other developmental outcomes. The field would benefit from a deeper understanding of the contribution of maternal and fetal genetics in regulating serotonin.

Because of low exposure prevalence and possible cofactors influencing susceptibility to ASD from SSRI exposure, any contribution of these medications to the increase in autism diagnoses over time is likely minimal. ${ }^{44}$ The reported results must be viewed in the context of the disorder they are used to treat and, particularly, with the risks associated with failure to treat the condition. Maternal depression during pregnancy has itself been linked to preterm birth, fetal growth restriction, and preeclampsia, ${ }^{45}$ as well as increased irritability in newborns and reduced activity and attentiveness compared with infants of nondepressed women. ${ }^{46}$ Moreover, gestational depression has been observed to affect DD independent of postpartum depression. ${ }^{47}$ Given these negative consequences, depression during pregnancy and the positive aspects of pharmacologic management present pregnant women and their doctors with complex treatment decisions. The benefits of treating depression with SSRIs during pregnancy should continue to be carefully weighed against any potential risk of harm. 


\section{REFERENCES}

1. Autism and Developmental Disabilities Monitoring Network Surveillance Year 2008 Principal Investigators; Centers for Disease Control and Prevention. Prevalence of autism spectrum disorders-Autism and Developmental Disabilities Monitoring Network, 14 sites, United States, 2008. MMWR Surveill Summ. 2012;61(3):1-19

2. Mulder EJ, Anderson GM, Kema IP, et al. Platelet serotonin levels in pervasive developmental disorders and mental retardation: diagnostic group differences, within-group distribution, and behavioral correlates. J Am Acad Child Adolesc Psychiatry. 2004:43(4):491-499

3. Whitaker-Azmitia PM. Behavioral and cellular consequences of increasing serotonergic activity during brain development: a role in autism? Int J Dev Neurosci. 2005; 23(1):75-83

4. Alwan S, Reefhuis J, Rasmussen SA, Friedman JM; National Birth Defects Prevention Study. Patterns of antidepressant medication use among pregnant women in a United States population. J Clin Pharmacol. 2011;51(2): 264-270

5. Whitaker-Azmitia PM. Serotonin and brain development: role in human developmental diseases. Brain Res Bull. 2001;56(5):479_ 485

6. Bonnin A, Goeden N, Chen K, et al. A transient placental source of serotonin for the fetal forebrain. Nature. 2011;472(7343): 347-350

7. Dufour-Rainfray D, Vourc'h P, Le Guisquet $\mathrm{AM}$, et al. Behavior and serotonergic disorders in rats exposed prenatally to valproate: a model for autism. Neurosci Lett. 2010;470 (1):55-59

8. Rayen I, van den Hove DL, Prickaerts J, Steinbusch HW, Pawluski JL. Fluoxetine during development reverses the effects of prenatal stress on depressive-like behavior and hippocampal neurogenesis in adolescence. PLOS ONE. 2011;6(9):e24003

9. Hendrick V, Stowe ZN, Altshuler LL, Hwang $S$, Lee $E$, Haynes D. Placental passage of antidepressant medications. Am J Psychiatry. 2003;160(5):993-996

10. Cool DR, Liebach FH, Ganapathy V. Interaction of fluoxetine with the human placental serotonin transporter. Biochem Pharmacol. 1990;40(9):2161-2167

11. Anderson GM, Czarkowski K, Ravski N, Epperson CN. Platelet serotonin in newborns and infants: ontogeny, heritability, and effect of in utero exposure to selective serotonin reuptake inhibitors. Pediatr Res. 2004;56(3):418-422
12. Rurak D, Lim K, Sanders A, Brain U, Riggs W, Oberlander TF. Third trimester fetal heart rate and Doppler middle cerebral artery blood flow velocity characteristics during prenatal selective serotonin reuptake inhibitor exposure. Pediatr Res. 2011;70(1): 96-101

13. Burstyn I, Wang X, Yasui Y, Sithole F, Zwaigenbaum L. Autism spectrum disorders and fetal hypoxia in a population-based cohort: accounting for missing exposures via Estimation-Maximization algorithm. BMC Med Res Methodol. 2011;11:2

14. Croen LA, Grether JK, Yoshida CK, Odouli R, Hendrick V. Antidepressant use during pregnancy and childhood autism spectrum disorders. Arch Gen Psychiatry. 2011;68 (11):1104-1112

15. Rai D, Lee BK, Dalman C, Golding J, Lewis G, Magnusson C. Parental depression, maternal antidepressant use during pregnancy, and risk of autism spectrum disorders: population based case-control study. BMJ. 2013;346(7907):f2059

16. Hviid A, Melbye M, Pasternak B. Use of selective serotonin reuptake inhibitors during pregnancy and risk of autism. $N$ Engl J Med. 2013;369(25):2406-2415

17. Hertz-Picciotto I, Croen LA, Hansen R, Jones CR, van de Water J, Pessah IN. The CHARGE study: an epidemiologic investigation of genetic and environmental factors contributing to autism. Environ Health Perspect. 2006;114(7):1119-1125

18. Rutter M, Le Couteur A, Lord C. Autism Diagnostic Interview-Revised. Los Angeles, CA: Western Psychological Services; 2005

19. Lord C, Rutter M, DiLavore PC, Risi S. Autism Diagnostic Observation Schedule. Los Angeles, CA: Western Psychological Services; 2007

20. Risi S, Lord C, Gotham K, et al. Combining information from multiple sources in the diagnosis of autism spectrum disorders. J Am Acad Child Adolesc Psychiatry. 2006; 45(9):1094-1103

21. Rutter M, Bailey A, Lord C. Social Communication Questionnaire. Los Angeles, CA: Western Psychological Services; 2003

22. Sparrow SS, Balla DA, Cicchetti DV. Vineland Adaptive Behavior Scales Interview Edition Expanded Form. Circle Pines, MN: American Guidance Service, Inc; 1984

23. Mullen EM. Mullen Scales of Early Learning. Circle Pines, MN: American Guidance Service, Inc; 1995

24. Rothman KJ, Greenland S, Lash TL. Validity in epidemiologic studies. In: Rothman KJ, Greenland S, Lash TL, eds. Modern Epide- miology. 3rd ed. Philadelphia, PA: Lippincott Williams \& Wilkins; 2008:128-147

25. Landis JR, Koch GG. The measurement of observer agreement for categorical data Biometrics. 1977;33(1):159-174

26. Bethea CL, Pecins-Thompson M, Schutzer WE, Gundlah C, Lu ZN. Ovarian steroids and serotonin neural function. Mol Neurobiol 1998;18(2):87-123

27. de Souza Silva MA, Mattern C, Topic B, Buddenberg TE, Huston JP. Dopaminergic and serotonergic activity in neostriatum and nucleus accumbens enhanced by intranasal administration of testosterone. Eur Neuropsychopharmacol. 2009;19(1):5363

28. Weiss LA, Abney M, Cook EH Jr, Ober C. Sexspecific genetic architecture of whole blood serotonin levels. Am J Hum Genet. 2005;76(1):33-41

29. Hohmann CF, Walker EM, Boylan CB, Blue ME. Neonatal serotonin depletion alters behavioral responses to spatial change and novelty. Brain Res. 2007;1139:163-177

30. Lisboa SF, Oliveira PE, Costa LC, Venâncio EJ, Moreira EG. Behavioral evaluation of male and female mice pups exposed to fluoxetine during pregnancy and lactation. Pharmacology. 2007;80(1):49-56

31. Pedersen LH, Henriksen TB, Olsen J. Fetal exposure to antidepressants and normal milestone development at 6 and 19 months of age. Pediatrics. 2010;125(3). Available at www.pediatrics.org/cgi/content/full/125/3/ e600

32. Jokiranta E, Brown AS, Heinimaa M, CheslackPostava K, Suominen A, Sourander A. Parental psychiatric disorders and autism spectrum disorders. Psychiatry Res. 2013;207(3):203211

33. Larson MJ, Miller K, Fleming KJ. Treatment with antidepressant medications in private health plans. Adm Policy Ment Health. 2007; 34(2):116-126

34. Newport DJ, Brennan PA, Green P, et al Maternal depression and medication ex posure during pregnancy: comparison of maternal retrospective recall to prospective documentation. BJOG. 2008;115(6): 681-688

35. Gentile S, Galbally M. Prenatal exposure to antidepressant medications and neurodevelopmental outcomes: a systematic review. J Affect Disord. 2011;128(1-2):1-9

36. Casper RC, Fleisher BE, Lee-Ancajas JC et al. Follow-up of children of depressed mothers exposed or not exposed to antidepressant drugs during pregnancy. J Pediatr. 2003;142(4):402-408 
37. Casper RC, Gilles AA, Fleisher BE, Baran J, Enns G, Lazzeroni LC. Length of prenatal exposure to selective serotonin reuptake inhibitor (SSRI) antidepressants: effects on neonatal adaptation and psychomotor development. Psychopharmacology (Berl). 2011;217(2):211-219

38. Rice F, Lewis A, Harold G, et al. Agreement between maternal report and antenatal records for a range of pre and peri-natal factors: the influence of maternal and child characteristics. Early Hum Dev. 2007;83(8): 497-504

39. Goodnick PJ, Goldstein BJ. Selective serotonin reuptake inhibitors in affective disorders-I. Basic pharmacology. J Psychopharmacol. 1998;12(3 suppl B):S5-S20

40. Preskorn SH. The use of biomarkers in psychiatric research: how serotonin transporter occupancy explains the doseresponse curves of SSRIs. I Psychiatr Pract. 2012;18(1):38-45

41. Rampono J, Simmer K, llett KF, et al. Placental transfer of SSRI and SNRI antidepressants and effects on the neonate. Pharmacopsychiatry. 2009;42(3):95-100

42. Harrington RA, Lee LC, Crum RM, Zimmerman AW, Hertz-Picciotto I. Serotonin hypothesis of autism: implications for selective serotonin reuptake inhibitor use during pregnancy. Autism Res. 2013;6(3):149-168

43. Velasquez JC, Goeden N, Bonnin A. Placental serotonin: implications for the developmental effects of SSRIs and maternal depression. Front Cell Neurosci. 2013;7:47

44. Schieve LA, Rice C, Devine 0, et al. Have secular changes in perinatal risk factors contributed to the recent autism prevalence increase? Development and application of a mathematical assessment model. Ann Epidemiol. 2011;21(12):930945

45. Bonari L, Pinto N, Ahn E, Einarson A, Steiner M, Koren G. Perinatal risks of untreated depression during pregnancy. Can J Psychiatry. 2004;49(11):726-735

46. Yonkers KA, Wisner KL, Stewart DE, et al. The management of depression during pregnancy: a report from the American Psychiatric Association and the American College of Obstetricians and Gynecologists. Obstet Gynecol. 2009;114(3):703-713

47. Deave T, Heron J, Evans J, Emond A. The impact of maternal depression in pregnancy on early child development. BJOG. 2008;115(8):1043-1051

(Continued from first page)

FINANCIAL DISCLOSURE: The authors have indicated they have no financial relationships relevant to this article to disclose.

FUNDING: This research was supported by US National Institute on Environmental Health Sciences grants P01-ES11269 and R01-ES015359, the MIND Institute, and Autism Speaks. Funded by the National Institutes of Health (NIH).

POTENTIAL CONFLICT OF INTEREST: The authors have indicated they have no potential conflicts of interest to disclose. 